\title{
El componente prosódico en el estudio de la intercomprensión románica y su posible aplicación didáctica
}

\section{Beatriz Hernández y Josefa Dorta}

\author{
(2) OpenEdition \\ Journals \\ Edición electrónica \\ URL: https://journals.openedition.org/cher/896 \\ DOI: $10.4000 /$ cher.896 \\ ISSN: 2803-5992 \\ Editor \\ Presses universitaires de Strasbourg
}

Edición impresa

Fecha de publicación: 12 diciembre 2019

Paginación: 83-99

ISBN: 979-10-344-0057-7

ISSN: 1968-035X

Referencia electrónica

Beatriz Hernández y Josefa Dorta, «El componente prosódico en el estudio de la intercomprensión románica y su posible aplicación didáctica», reCHERches [En línea], 23 | 2019, Publicado el 27 septiembre 2021, consultado el 22 noviembre 2021. URL: http://journals.openedition.org/cher/896 ; DOI: https://doi.org/10.4000/cher.896 


\title{
El componente prosódico en el estudio de la intercomprensión románica y su posible aplicación didáctica*
}

\author{
BeAtriz Hernández \\ JOSEFA DORTA ${ }^{1}$
}

\begin{abstract}
$E^{l}$ 1 diseño de un corpus experimental en el proyecto AMPER (Atlas Multimedia de Prosodia del Espacio Románico $)^{2}$ tiene como objetivo poder comparar las distintas lenguas y variedades románicas a partir de estructuras sintácticas y acentuales idénticas o similares. Así, contamos ya con diversos trabajos en los que se comparan patrones entonativos de diferentes dominios románicos como, por ejemplo, el francés y el portugués (Zerling y Moutinho 2002), el portugués e italiano (Moutinho et al. 2004, Interlandi et al. 2007), el español y el asturiano (Dorta y Muñiz Cachón 2009), el portugués y el gallego (Fernández Rei et al. 2014), el catalán y el castellano (Romera Barrios et al. 2009), el friulano, italiano, castellano y catalán (Fernández Planas et al. 2013), o el catalán, gallego y español (Martínez Celdrán et al. 2006). De la misma manera, se han comparado diferentes variedades de una misma lengua como, por ejemplo, del portugués continental (Moutinho et al. 2005), del italiano (Avolio y Romano 2007), del rumano (Turculet y Bleortu 2013), del friulano (Roseano y Fernández Planas 2013), del asturiano (Alvarellos Pedrero et al. 2011), o del español, lengua esta en la que se han contrastado, por ejemplo, variedades insulares y americanas
\end{abstract}

* Este trabajo se ha realizado en el marco del proyecto Estudio comparativo de la entonación y del acento en zonas fronterizas del español (FFI2014-52716-P), proyecto de $\mathrm{I}+\mathrm{D}$ del programa estatal de fomento de la investigación científica y técnica de excelencia, subprograma estatal de generación del conocimiento del Ministerio de Economía y Competitividad de España (2015-2017, convocatoria de 2014).

1 Beatriz Hernández, Académie d'Aix-Marseille, ORCID: 0000-0002-0140-0319 y Josefa Dorta, Universidad de La Laguna, ORCID: 0000-0002-5204-8968

2 Este proyecto fue concebido a finales del siglo $\mathrm{xx}$ en el Centre de Dialectologie de l'Université Stendhal-Grenoble 3 con el objetivo general de realizar un estudio geoprosódico de las lenguas y variedades románicas de Europa y América (Contini et al. 2002). 
(v. gr. Dorta ed. 2013, 2018), variedades peninsulares e insulares (Fernández Planas et al. 2013, 2015) o, incluso, microvariedades insulares (Dorta y Díaz 2013) $)^{3}$.

La metodología del proyecto AMPER permite realizar la comparación de los patrones entonativos de las diferentes lenguas o variedades teniendo en cuenta los datos fonéticos del análisis realizado ${ }^{4}$ pero, también, a partir de la percepción de oraciones sintetizadas. En este caso, se utilizan audios que reproducen solo la prosodia de las oraciones eliminando toda referencia al contenido léxicosemántico de las mismas. De esta manera, se han publicado ya algunos estudios perceptivos (Van Oosterzee 2005, Fernández Pérez-Terán et al. 2007, Martínez Celdrán et al. 2007, Mora et al. 2008, Romera Barrios et al. 2009, Dorta y Díaz 2013, 2014) en los que se han extraído conclusiones interesantes como, por ejemplo, que los finales descendentes se asocian a la modalidad declarativa, por lo que los auditores identifican mejor esta modalidad que la interrogativa, a pesar de que el reconocimiento de ambas modalidades no sea muy distante $(v$. gr. Martínez Celdrán et al. 2006, Dorta y Díaz 2013); asimismo, que cuando se perciben lenguas diferentes o variedades de una misma lengua, los estímulos sintetizados de la modalidad lingüística del auditor son los mejor reconocidos; no obstante, la intercomprensión entre lenguas románicas (Martínez Celdrán et al. 2006) o entre variedades de una misma lengua (Dorta y Díaz 2013) es muy buena, a pesar de que los estudios se basen en la audición de frases sintetizadas en lugar de emisiones normales. Teniendo en cuenta el tipo de acento agudo, llano o esdrújulo que abre y cierra cada uno de los estímulos, en un estudio en el que se contrastó el canario y el cubano (Dorta y Díaz 2013: 50-51) se llegó a la siguiente conclusión:

en las interrogativas, exceptuando las combinaciones con mayor índice de error, esto es, aguda-esdrújula $(57,14 \%)$ y llana-esdrújula $(66,67 \%)$, el resto de los estímulos tuvo un nivel de reconocimiento alto pues el porcentaje se sitúa a partir del 71,43\% (para los cubanos) y 66,67\% (para los canarios). En los estímulos declarativos, las combinaciones peor reconocidas fueron aguda-llana, llanaaguda, esdrújula-esdrújula, con el mismo índice de errores $(28,57 \%)$, cuando se trató de estímulos cubanos y la combinación llana-esdrújula $(38,1 \%)$ cuando fueron estímulos canarios. Salvo estos casos, los umbrales de reconocimiento se sitúan entre el $71 \%$ y $85 \%$, en los estímulos cubanos y entre el $61 \%$ y el $100 \%$, en los canarios.

En definitiva, en los estudios mencionados, ya sea en los que se basan en datos acústicos como en la percepción de la entonación, se trata de comprobar

3 Para consultar más referencias bibliográficas, véase «Bibliographie autour du projet AMPER» en <http://dialecto.u-grenoble3.fr/AMPER/pub.htm>; referencias del grupo de investigación al que pertenecen las autoras de este trabajo en $<$ http://ampercan. webs.ull.es/>.

4 El proyecto AMPER es eminentemente de naturaleza fonética, pero no impide que los resultados sean estudiados desde una perspectiva fonológica, como se ha hecho ya en diversos trabajos (v. gr. Dorta ed. 2013, 2018). 
el grado de proximidad o distancia prosódica entre las lenguas y variedades románicas con el fin último de observar si existe 'intercomprensión románica', es decir, comprensión recíproca entre hablantes del espacio lingüístico románico. El parentesco lingüístico, base de estos estudios, ha sido utilizado como un recurso didáctico en la enseñanza de lenguas románicas en proyectos centrados preferentemente en la comunicación escrita (Dabène 1975, Blanche-Benveniste et al. 1997). Más reciente es el interés, en esta materia, por la comprensión del discurso oral a partir de documentos sonoros o audiovisuales, si bien se ha relegado la importancia del aspecto prosódico. De ahí la importancia de que el proyecto internacional AMPER introduzca un enfoque fonético-perceptivo en el estudio de la intercomprensión atendiendo a la entonación y partiendo del principio de proximidad prosódica entre las distintas lenguas románicas.

El objetivo de nuestro trabajo, enmarcado en el referido proyecto, es realizar un estudio perceptivo a partir de estímulos sintetizados de cinco lenguas románicas: francés, rumano, italiano, portugués y español. Teniendo en cuenta afirmaciones como «Sembla que els oients tenen més facilitat per reconèixer la modalitat i també el grau de "normalitat" de les oracions a partir del seu mot final que de l'inicial» [Parece que los oyentes tienen más facilidad para reconocer la modalidad así como el grado de "normalidad" de las oraciones a partir de la palabra final que de la inicial] (Martínez Celdrán et al. 2006: 18), pretendemos observar la relevancia del núcleo entonativo en la percepción de estas lenguas con el propósito de dar respuesta a varios interrogantes: ¿cuál de estas lenguas es mejor reconocida por los «romanófonos»?, ¿qué modalidad es mejor reconocida?, ¿interviene la estructura acentual en el grado de reconocimiento?, y, por último, ¿puede hablarse, como en el nivel léxico, de «transparencia prosódica» entre lenguas de la misma familia ${ }^{5}$ ?

\section{Metodología}

\section{Corpus y variables consideradas}

Los archivos sintetizados, sin contenido léxico-semántico, presentados a los auditores fueron obtenidos con el programa AMPER 2006 (Brezmes Alonso 2007) ${ }^{6}$ a partir del análisis de un corpus de oraciones del tipo SVO (Tabla 1). En la configuración del corpus se tuvieron en cuenta distintas variables: la variedad románica, la modalidad oracional (declarativa e interrogativa) y la estructura acentual (oxítona, paroxítona y/o proparoxítona) de los enunciados. Las lenguas románicas elegidas fueron: francés (Marsella), rumano (Cluj, en la región de Transilvania), italiano (Milán), portugués (Lisboa) y español. De esta

5 Jamet (2007) habla de "la transparence sonore du lexique» y establece toda una gradación de palabras transparentes, semi-transparentes, semi-opacas y opacas entre lenguas emparentadas.

6 El programa lo ha realizado AMPER Asturias con rutinas de Matlab. La licencia de Matlab en el Laboratorio de Fonética de la ULL es la no 256105. Véase el replanteamiento de las nuevas rutinas en López Bobo et al. (2007). 
última lengua elegimos estímulos de dos variedades: el canario (La Laguna) y el castellano (Zaragoza), teniendo en cuenta la existencia de dos patrones interrogativos característicos de ambas variedades, esto es, el circunflejo (Dorta ed. 2013) y el ascendente, descrito ampliamente en la bibliografía (Fernández Planas et al. 2013). En lo que respecta a la producción, las oraciones fueron emitidas por seis mujeres cuya entonación se considera suficientemente representativa de sus variedades respectivas.

Tabla 1: Corpus sintetizado para el test.

\begin{tabular}{|l|l|l|}
\hline \multirow{2}{*}{ Francés } & \multicolumn{2}{|c|}{ Corpus } \\
& $\begin{array}{l}\text { Le saxophone touche la pauvreté./? } \\
\text { Le saxophone touche le xylophone./? }\end{array}$ & $\begin{array}{l}\text { SN llano - SP agudo } \\
\text { SN llano - SP llano }\end{array}$ \\
\hline Rumano & $\begin{array}{l}\text { Nevasta vede un căpitan./? } \\
\text { Nevasta vede nevasta./? } \\
\text { Nevasta vede pasărea./? }\end{array}$ & $\begin{array}{l}\text { SN llano - SP agudo } \\
\text { SN llano - SP llano }\end{array}$ \\
\hline Italiano & La papera mangia la maracà./? & SN esdrújulo - SP agudo \\
& La papera mangia la patata./? & SN esdrújulo - SP llano \\
& La papera mangia la fragola./? & SN esdrújulo - SP esdrújulo \\
\hline Portugués & O Toneca toca no capataz./? & SN llano - SP agudo \\
& O Toneca toca no Toneca./? & SN llano - SP llano \\
& O Toneca toca no pássaro./? & SN llano - SP esdrújulo \\
\hline Español & La guitarra se toca con obsesión./? & SN llano - SP agudo \\
& La guitarra se toca con paciencia./? & SN llano - SP llano \\
& La guitarra se toca con pánico./? & SN llano - SP esdrújulo \\
\hline
\end{tabular}

\section{Test de percepción y auditores}

Para el diseño y la configuración del test se eligió el software libre TP (v. 3.1) (Rodrigues dos Santos et al. 2013) que permite realizar test de identificación y de respuesta cerrada: se ofrece un estímulo cada vez y el auditor (juez), tras escucharlo varias veces, si lo desea, debe elegir una opción (declarativa o interrogativa) para identificar el estímulo que escucha respondiendo a la cuestión «¿El estímulo que oyes corresponde a una declarativa o a una interrogativa?». El conjunto de estímulos se repite tres veces, mezclados aleatoriamente, por lo que no se reproducen en el mismo orden en cada repetición. De este modo, podemos observar si hay o no respuestas al azar.

El test estuvo integrado por 34 estímulos sintetizados manipulados, puesto que se suprimió el sintagma nominal con el objetivo de determinar hasta qué punto el núcleo entonativo permite interpretar los estímulos correctamente ${ }^{7}$. Por tanto, estos corresponden a oraciones con final agudo, llano y esdrújulo ${ }^{8}$. Al

7 En realidad, cada estímulo corresponde a la síntesis del SV+SPrep, pero ha de tenerse en cuenta que el núcleo del SV es invariable en cuanto al acento, mientras que el núcleo del SPrep es agudo, llano o esdrújulo.

8 En el caso del francés no se consideró el final esdrújulo. Sin embargo, la elección de la variedad marsellesa nos permitió obtener estructuras llanas gracias a la realización de schwas finales característica de ciertas hablas meridionales. 
repetirse los estímulos del test tres veces, el conjunto de respuestas fue de 102 . El programa guarda automáticamente cada una de las respuestas en una hoja excel donde identifica al juez y muestra diferentes datos (fecha de realización, tiempo empleado en la realización, porcentajes de aciertos y errores, etc.).

El test fue presentado en un aula de informática en la que los estímulos fueron escuchados a través de cascos en un ordenador independiente. Un primer grupo de auditores fue de 67 hablantes nativos del español (46 mujeres y 21 hombres, todos estudiantes de la Universidad de La Laguna, islas Canarias) con edades comprendidas entre 19 y 25 años. Un segundo grupo estuvo formado por 30 mujeres nativas del francés, estudiantes de español en la Universidad de Toulon (niveles B1-B2 del MCERL) que, presumiblemente, permitirán establecer distintos grados en la identificación de las lenguas románicas al introducir como factor nuevo el conocimiento de una LE además de la suya propia9 .

\section{Resultados}

El test, como se ha dicho, incluyó estímulos sintetizados del SV+SPrep por lo que el reconocimiento se fundamenta, sobre todo, en la percepción de la parte nuclear. En las figuras 1-3 se ilustra el patrón tonal correspondiente a las oraciones con final llano.



9 En un próximo trabajo compararemos los resultados con los obtenidos por un tercer grupo de auditores formado por hablantes nativos del francés sin conocimientos de español. 



Figuras 1-3: Oraciones con final llano.

Como puede verse en la figura 1, todas las lenguas coinciden en presentar un patrón tonal descendente en las declarativas; las interrogativas, en cambio, diferencian dos grupos de lenguas: el zaragozano, el francés y el italiano (figura 2) terminan con un tonema ascendente; en cambio, el tinerfeño, el portugués y el rumano (figura 3) acaban en un final claramente circunflejo ${ }^{10}$. El test de percepción nos permitirá determinar hasta qué punto las diferencias ilustradas constituyen o no barreras prosódicas que den respuesta a los interrogantes planteados en la introducción.

$10 \mathrm{Ha}$ de tenerse en cuenta que solo hemos ilustrado con un ejemplo el contorno tonal de las oraciones cuya síntesis se ha presentado a los auditores. Por otra parte, en lenguas como el italiano y el rumano hay mucha variedad dialectal y, por ello, las configuraciones tonales no presentan el mismo tonema en todas. Por último, teniendo en cuenta que nuestro corpus se ha realizado siguiendo la metodología de AMPER, remitimos a Paolo Mairano (2011) para conocer con más detalle y datos las configuraciones de las declarativas e interrogativas en las diferentes lenguas románicas. 
La figura 4 evidencia que los porcentajes de aciertos y de errores en los auditores españoles de ambos sexos y en las auditoras francesas son prácticamente los mismos, por lo que el promedio general de reconocimiento (67\%) es bueno (17 puntos por encima del $50 \%$ ) teniendo en cuenta las características de los estímulos y que el índice de aciertos duplica al de errores (33\% de promedio).



Figura 4: Promedio general de aciertos y errores de estímulos sintetizados de oraciones SVO con final agudo, llano y esdrújulo. $\mathrm{ME}=$ Mujeres españolas; $\mathrm{MF}=$ Mujeres francesas y $\mathrm{HE}=\mathrm{Hombres}$ españoles.

En lo que respecta al reconocimiento de las diferentes lenguas, el francés y el español se sitúan a la cabeza en la escala de mejor reconocimiento con un promedio general de aciertos, teniendo en cuenta el conjunto de los auditores, del $79 \%$ y $71 \%$, respectivamente. Las peor reconocidas son el italiano, el portugués y el rumano con porcentajes próximos entre sí ( $62 \%$, 60\% y $58 \%$, respectivamente). La figura 5 muestra el promedio de reconocimiento en cada grupo de auditores.

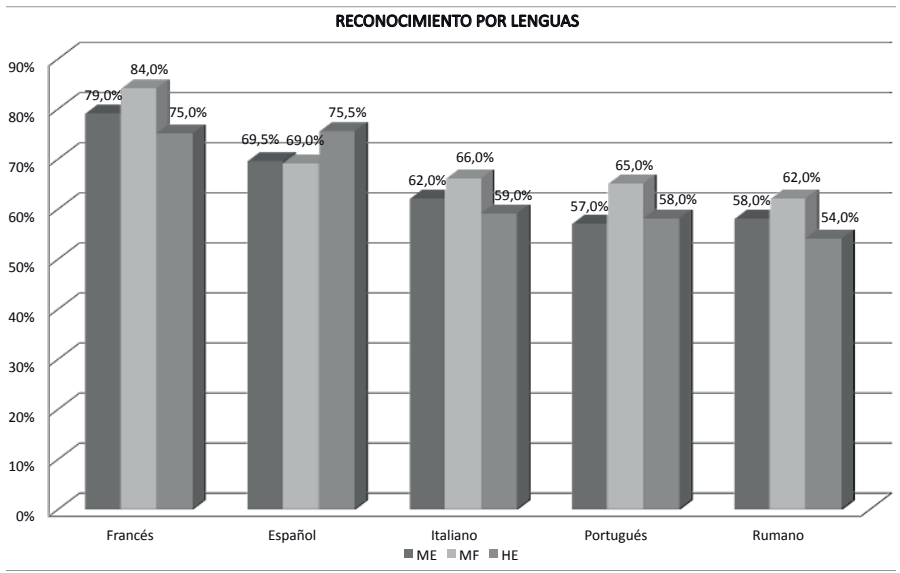

Figura 5: Promedio de aciertos por lengua. 
De estos resultados destaca el hecho de que el francés sea la lengua mejor reconocida, tanto por las mujeres francesas como por las españolas, aunque de manera más notable por las que lo tienen como lengua materna ( $84 \%$ y $79 \%$, respectivamente). El español aparece en segundo lugar, con porcentajes prácticamente iguales en las mujeres, aunque la diferencia con respecto a la primera posición es más destacada para las auditoras francesas ( $15 \%$ frente al 9,5\% de las españolas). En esta segunda posición de mejor reconocimiento, el porcentaje de los hombres aventaja al de las mujeres $(75,5 \%)$ y es prácticamente el mismo que el que se da en el reconocimiento del francés. En las tres lenguas restantes, el índice de aciertos de las francesas es siempre superior al de los auditores españoles.

En el español, como se ha dicho, hemos analizado dos variedades caracterizadas por diferente tonema final (ascendente vs circunflejo). El reconocimiento de ambas variedades fue muy similar y alto, pero el zaragozano es ligeramente mejor reconocido que el canario tanto por los españoles $(73,5 \%$ vs $71,5 \%$, respectivamente), como por las mujeres francesas ( $72 \%$ vs $66 \%$, respectivamente).

En lo que respecta al reconocimiento de la modalidad, el promedio de aciertos del conjunto de los auditores sitúa a las declarativas un $14 \%$ por encima de las interrogativas ( $74 \%$ y $60 \%$, respectivamente). Si observamos la figura 6 puede verse que los porcentajes de aciertos son muy próximos en los tres grupos de auditores, pero algo más en las declarativas.

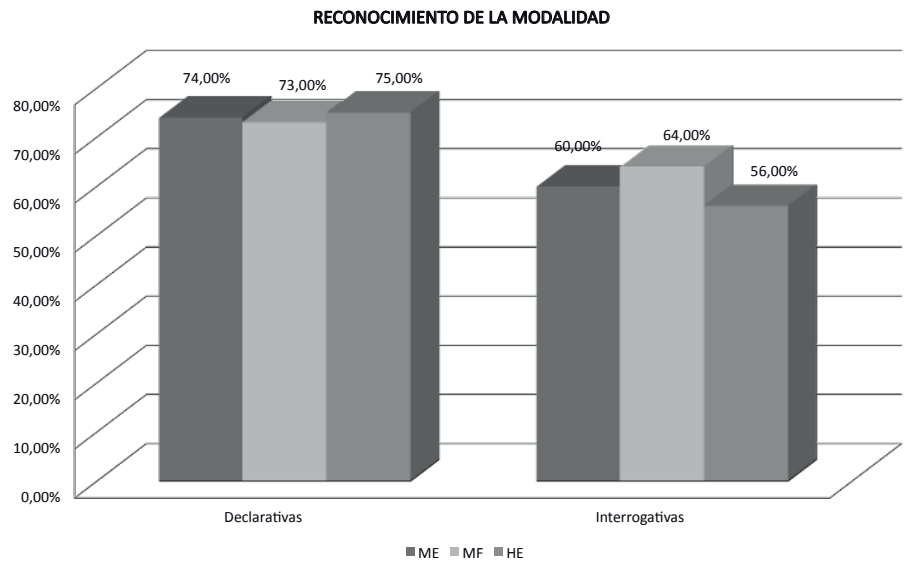

Figura 6: Promedio de aciertos por modalidad.

Por último, se observaron importantes diferencias en el índice de reconocimiento en función del tipo de acento nuclear. En las declarativas, los estímulos mejor reconocidos por los auditores españoles, con un porcentaje superior al 90\%, fueron los correspondientes a oraciones con final llano y agudo del francés y los esdrújulos del portugués (figura 7). Las auditoras francesas coinciden con los españoles (figura 8), excepto en los finales llanos que, no 
obstante, tienen un porcentaje de reconocimiento muy próximo (86\%). Los estímulos peor reconocidos por los españoles, con un porcentaje inferior al $50 \%$, fueron los llanos y esdrújulos del rumano. Estos últimos son, asimismo, los peor reconocidos por las auditoras francesas, aunque con un porcentaje algo más elevado (56\%). Si atendemos a qué tipo de final fue el mejor reconocido en cada lengua, observamos que en las declarativas del francés ambos grupos de auditores reconocen los finales agudos y llanos con porcentajes muy altos y similares; en las del portugués y el español, en cambio, son las de final esdrújulo las mejor reconocidas. En las declarativas italianas, los auditores franceses reconocen mejor los estímulos con final llano, mientras que los españoles obtuvieron mayor índice de aciertos -con un porcentaje similar- en los de final agudo, final este que es, asimismo, el mejor reconocido en rumano por los dos grupos de auditores.
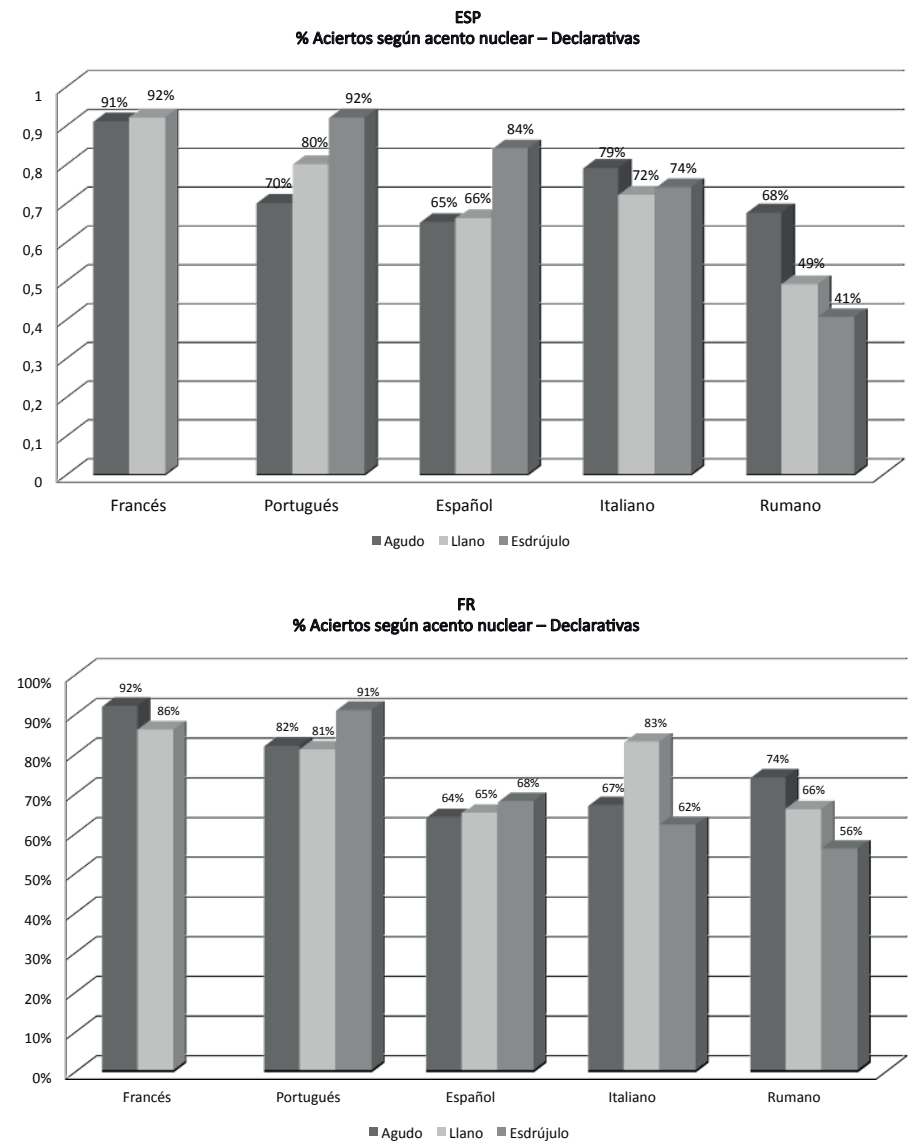

Figuras 7 y 8: Promedio de aciertos según el acento nuclear en declarativas 
En lo que respecta a las interrogativas, los dos grupos de auditores coinciden en que los estímulos mejor reconocidos, con porcentajes superiores al $80 \%$, son los agudos del rumano y los llanos del español y del francés (figuras $9 \mathrm{y}$ 10). Asimismo, coinciden en que los esdrújulos del rumano tienen un índice muy bajo de reconocimiento, en torno al $20 \%$, como sucede en los agudos del portugués por parte de los auditores españoles. Si consideramos el mejor reconocimiento en cada lengua, los dos grupos coinciden plenamente, puesto que, en primer lugar, el rumano es la única lengua donde los finales agudos fueron los mejor reconocidos, con diferencias muy destacadas respecto de los otros dos acentos, sobre todo en relación con los esdrújulos ${ }^{11}$. Por otra parte, en español, francés e italiano destaca el reconocimiento de los estímulos con final llano, mientras que en portugués sobresalen los de final esdrújulo.
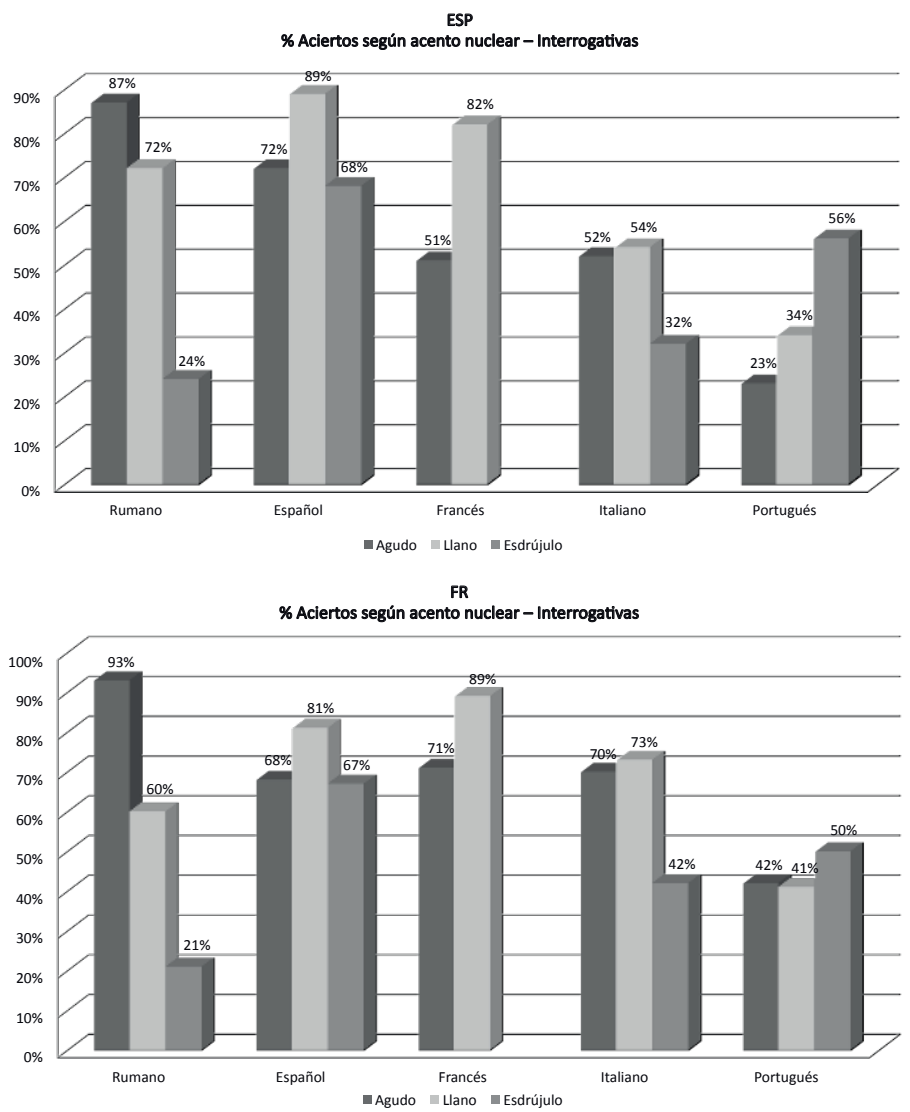

Figuras 9 y 10: Promedio de aciertos según el acento nuclear en interrogativas

11 En ambas modalidades los estímulos con final esdrújulo del rumano fueron los peor reconocidos, con porcentajes de acierto más bajos en las interrogativas. 
Los resultados comentados evidencian que en las declarativas los jueces españoles, a diferencia del auditorio francés, no reconocen los finales de su propia lengua de manera destacada respecto de las otras lenguas románicas ${ }^{12}$. Por el contrario, en las interrogativas los dos grupos de auditores destacan el reconocimiento de los finales de sus propias lenguas, sobre todo el de los llanos. Este hecho es explicable en español, puesto que este acento es el más representativo de esta lengua; en cambio, si tenemos en cuenta esta circunstancia, se esperaría que en francés lo fuera el acento agudo.

\section{Aplicación didáctica}

Después de una exhaustiva revisión bibliográfica relacionada con la didáctica de la lengua extranjera, Cortés Moreno (2001: 138) concluye:

[...] resulta evidente que a la entonología aplicada a la didáctica del E/LE todavía le queda un largo camino por recorrer hasta alcanzar un puesto en consonancia con el peso específico de la entonación en la comunicación.

Puede decirse que, aún hoy, el panorama sigue siendo prácticamente el mismo, por lo que intentaremos ofrecer en este último apartado algunas líneas de actuación que puedan orientar el diseño y la confección de nuevas actividades para la integración del suprasegmento entonativo en la clase de E/LE.

Por otra parte, estudios previos (Cortés Moreno 2005, Sensui 2015) han demostrado que los estudiantes de español tienen dificultades de producción y de percepción con algunos patrones acentuales y entonativos, lo que los lleva muchas veces a identificar incorrectamente la modalidad de frase. Así, en un trabajo reciente (Martín Gómez, Dorta Luis y Sensui 2017) se investigó si un grupo de japoneses estudiantes de E/LE y otro sin conocimientos de esta lengua distinguen correctamente los patrones ascendente $/ \mathrm{H}^{*} /$ (variedad septentrional del español) y circunflejo $/ \mathrm{H}^{*} \mathrm{~L} \% /$ de algunas variedades hispanohablantes (como el canario). Los resultados mostraron que los dos grupos reconocen muy bien el primer patrón de las interrogativas, que es el que usan en su lengua materna para la misma finalidad, pero no sucede lo mismo con el segundo. Por ello, se concluyó (2017: 24-25):

sería de gran importancia entrenar a los estudiantes japoneses de español, así como a otros estudiantes extranjeros, en el reconocimiento de estos dos patrones interrogativos usados ampliamente en el español, enseñando a los alumnos cómo discriminar entre las marcas de acento y modalidad para que puedan percibir y producir correctamente tanto el acento de la última palabra de las oraciones como cualquiera de los dos patrones interrogativos. En caso contrario, estos alumnos se verían en graves dificultades comunicativas, como hemos descrito, sobre todo al intentar comunicarse con algún hablante de las variedades que tienen como principal patrón interrogativo el circunflejo.

12 Las auditoras francesas sitúan también el reconocimiento de los finales del portugués en un lugar muy destacado en relación con el resto de las lenguas románicas. 
En consonancia con esta conclusión, consideramos que la explotación didáctica de los resultados obtenidos en el presente trabajo puede ser de especial interés para la enseñanza «explícita» de la entonación con aprendientes de distinta procedencia ${ }^{13}$. Reconocida la suficiencia significativa del núcleo, resulta evidente que los archivos sintetizados, sin contenido léxico-semántico, aquí utilizados podrían convertirse en un recurso didáctico realmente útil para la enseñanza-aprendizaje de las estructuras prosódicas básicas no solo del español sino de cualquiera de las lenguas estudiadas, pasando por el reconocimiento de la información lingüística en una primera fase antes de abordar la producción oral de dichos patrones. En este sentido, sería interesante comprobar en el aula hasta qué punto los patrones melódicos de la L1 pueden favorecer o interferir el aprendizaje de la LE, con el fin de adaptar el diseño de las actividades destinadas a la enseñanza de la entonación, reduciendo, cuanto sea posible, la distancia entre la prosodia de la interlengua y la de la lengua meta, lo que sin duda contribuiría a atenuar el llamado «acento extranjero».

Si el enfoque comunicativo y la «perspectiva accional» del MCERL abogan por la recreación de situaciones comunicativas reales en el aula, la integración de la entonación resulta fundamental para una mayor naturalidad de las emisiones. Ahora bien, nuestra propuesta didáctica no pasaría solo por el entrenamiento a la discriminación de la modalidad oracional y la (re)producción de patrones entonativos declarativos y/o interrogativos en actividades de interacción, sino que podría extenderse a una "comunicación multimodal», de manera que el hablante sea capaz de asociar emociones y entonemas aprendidos en la clase de E/LE.

Por último, por sus implicaciones con la intención comunicativa, consideramos que otro de los objetivos fundamentales para la adquisición de las competencias orales básicas debería centrarse en la ironía verbal, siendo la prosodia -además de la información del contexto situacional o de la gestualidad- uno de sus principales marcadores a través del aumento de la frecuencia fundamental, la duración y la intensidad, entre otros índices acústicos. De este modo, podrían proponerse actividades de reconocimiento de enunciados irónicos frente a enunciados neutros antes de confrontar al alumno a situaciones comunicativas donde la percepción, así como la producción, de la «ironicidad» resulten indispensables.

\section{Discusión}

El presente estudio ratifica uno de los resultados obtenidos en trabajos anteriores en los que se han utilizado estímulos sintetizados para el reconocimiento: se confirma que los auditores reconocen mejor la modalidad declarativa que la interrogativa (Martínez Celdrán et al. 2006, Dorta y Díaz 2013).

13 Para una defensa de la enseñanza explícita del componente prosódico en la clase de E/LE véase Orta Gracia (2009). 
Teniendo en cuenta que los estímulos presentados en el test son sintetizados y, además, que no corresponden a la emisión completa de la oración, puesto que se eliminó la parte inicial, podemos decir que la intercomprensión entre las lenguas románicas es buena (Martínez Celdrán et al. 2006), aunque unas son mejor reconocidas que otras (figura 5). El presente estudio no corrobora del todo que, cuando se perciben lenguas diferentes o variedades de una misma lengua, los estímulos sintetizados de la modalidad lingüística del auditor sean los mejor reconocidos (Martínez Celdrán et al. 2006, Dorta y Díaz 2013), pues nuestros resultados evidenciaron que el francés es la lengua mejor reconocida, tanto por las mujeres francesas como por las españolas, no así por los hombres.

\section{Conclusiones}


los casos. Por tanto, el test reveló que, efectivamente, la parte nuclear es suficiente para el reconocimiento de la modalidad, si bien resulta evidente que la inclusión del segmento inicial contribuye a una mejora de los resultados. En el reconocimiento por lenguas, el francés y el español, por este orden, se mantienen como lenguas mejor reconocidas, seguidas del italiano, portugués y rumano que, con porcentajes no muy distantes entre sí, se sitúan a la cola en la escala de aciertos. El reconocimiento por modalidad nos muestra un promedio de aciertos más elevado en las declarativas, más fácilmente reconocibles gracias al final descendente característico de esta modalidad que comparten todas las lenguas.

$2^{\text {a) }}$ Teniendo en cuenta el sexo de los auditores, el porcentaje general de reconocimiento no varía prácticamente de hombres a mujeres, presentando ambos la misma escala de mayor a menor índice de aciertos. Las diferencias se dan en la modalidad interrogativa, con ligera ventaja del auditorio femenino, mientras que los estímulos declarativos fueron igualmente reconocidos por ambos sexos.

$3^{\text {a) }}$ Teniendo en cuenta la lengua materna del auditorio, españolas y francesas presentan porcentajes generales de aciertos y errores muy próximos entre sí. Esta igualdad se deshace si atendemos al reconocimiento por modalidad, con ligera ventaja de las oyentes francesas en la percepción de las interrogativas. De las lenguas escuchadas, ambos grupos coinciden en el mejor reconocimiento del francés y en el peor del portugués y el rumano, si bien los porcentajes de aciertos de las oyentes francesas son mayores en todos los casos.

$4^{\text {a) }}$ Por último, se observó que el tipo de acento nuclear condiciona el reconocimiento según la lengua y la modalidad. Así, independientemente del sexo y del origen de los auditores, en la modalidad declarativa, los finales agudos fueron generalmente mejor reconocidos en rumano y, junto a los finales llanos, en francés e italiano ${ }^{14}$; los finales esdrújulos fueron mejor reconocidos en español

14 Se exceptúa solo que los auditores españoles reconocen algo mejor los estímulos esdrújulos que los llanos en italiano (figura 7). 
y portugués. En la modalidad interrogativa, los finales agudos presentaron mejor reconocimiento en rumano, los finales esdrújulos en portugués y los finales llanos en español, francés e italiano. Estas tres lenguas -el español en su variedad zaragozana- son, precisamente, las que presentan un tonema final ascendente, por lo que se confirma la mejor recepción del llamado "patrón europeo» para las interrogativas incluso cuando no es característico de la propia variedad del oyente.


respuesta a las preguntas que nos hacíamos en la introducción en relación con la lengua mejor reconocida por los «romanófonos», la modalidad oracional con menor índice de error o la incidencia de la estructura acentual en el grado de reconocimiento. Por último, nos preguntábamos si podría hablarse de «transparencia prosódica» entre lenguas de la misma familia. Nuestra respuesta, a falta de otros estudios con auditores nativos de otras lenguas, es afirmativa. Así, decimos que el patrón final ascendente de las interrogativas peninsulares resultó transparente cuando los auditores franceses supieron interpretarlas en un elevado porcentaje (93\% frente al 51,7\% de las interrogativas canarias con final circunflejo). A la inversa, la pretendida «opacidad» de las interrogativas con final llano del francés no resultó ser un obstáculo para la percepción; al contrario, fueron mejor percibidas que las de final agudo típicamente características del francés estándar.

$6^{\text {a) }}$ Este trabajo propone la introducción del componente entonativo tanto en el estudio como en la práctica de la intercomprensión con fines didácticos para un dominio adecuado de las competencias orales de recepción y de producción, exigencia ineludible si el alumno aspira a convertirse en un verdadero "agente social» a través del uso de la LE.

\section{Bibliografía}

Alvarellos Pedrero M., Muñiz Cachón C., Díaz Gómez L. y González Rodríguez R., 2011, «La entonación en las variedades lingüísticas de Asturias: estudio contrastivo», RILI, 17/1 (9), p. 111-120.

Avolio F. y Romano A., 2007, «Aproximación á entoación dos enunciados declarativos e interrogativos en duas áreas dialectais da Italia da centromeridional (Abruzzo e Basilicata)», in González González M., Fernández Rei E. y González Rei B. (eds.), Actas del III Congreso Internacional de Fonética Experimental, Santiago de Compostela, Xunta de Galicia, p. 89-100.

Blanche-Benveniste C. (dir.), 1997, Eurom4. Méthode d'enseignement simultané des langues romanes, Florencia, La Nuova Italia.

Brezmes Alonso D., 2007, «Desarrollo de una aplicación software para el análisis de características fundamentales de la voz», Proyecto de fin de carrera, Oviedo, Ingeniería de Telecomunicación, Universidad de Oviedo. 
Contini M., Lai J-P., Romano A. y Roullet S., 2002, «Vers un Atlas prosodique parlant des variétés romanes», in Bouvier J-C., Gourc, J. y Pic F. (eds.), Mélanges offerts à Xavier Ravier, Sempre los camps auràn segadas resurgantas, Toulouse, Université de Toulouse-Le Mirail FRAMESPA, «Collection Méridiennes», p. 73-85.

Cortés Moreno M., 2001, «El papel de la prosodia en la enseñanza de la lengua extranjera. Una revisión de materiales didácticos», Lenguaje y textos, 17, p. 127-144.

Cortés Moreno M., 2005, «Análisis experimental del aprendizaje de la acentuación y la entonación española por parte de hablantes nativos de chino», Phonica, 1, p. 1-25.

Dabène L., 1975, «L'enseignement de l'espagnol aux francophones: pour une didactique des langues voisines», Langages, 39, p. 51-64.

Dorta J. (ed.), 2013, Estudio comparativo preliminar de la entonación de Canarias, Cuba y Venezuela, Madrid-Santa Cruz de Tenerife, La Página ediciones S/L, «Colección Universidad».

Dorta J. (ed.), 2018, La entonación declarativa e interrogativa en cinco zonas fronterizas del español: Canarias, Cuba, Venezuela, Colombia y San Antonio de Texas, Peter Lang Edition, Studien zur Romanischen Sprachwissenschaft und Interkulturellen Kommunikation, Herausgegeben von Gerd Wotjak.

Dorta J. y Díaz Ch., 2013, «Proximidad perceptivo-entonativa en dos variedades atlánticas: el caso canario-cubano», Lengua y Habla, 17, p. 34-54.

Dorta J. y Díaz Ch., 2014, «Reconocimiento perceptivo de patrones interrogativos coexistentes en Canarias», Fortunatae, Homenaje al Dr. Fremiot Hernández González, p. 115-127.

Dorta J. y Muñiz Cachón M. C., 2009, «La entonación de las interrogativas en el español de Canarias y en asturiano», in Beltrán Tejera E., Alfonso Carrillo J., García Gallo A. y Rodríguez Delgado O. (eds.), Homenaje al Profesor Dr. Wolfredo Wildpret de la Torre, Instituto de Estudios Canarios, La Laguna. Monografía LXXVIII, p. 809-821.

Fernández Pérez-Terán F., Dorta J., Ramos D. y García Riverón R., 2007, «La interrogativa absoluta en el español de Canarias y Cuba: estudio perceptivo», in Dorta J. (ed.), La prosodia en el ámbito lingüístico románico, Madrid-Santa Cruz de Tenerife, La Página Ediciones, «Colección Universidad», p. 371-387.

Fernández Planas A. M. a, Dorta J., Roseano P., Díaz Ch., Elvira García W., Martín Gómez J. A. y Martínez Celdrán E., 2015, «Distancia y proximidad prosódica entre algunas variedades del español: un estudio dialectométrico a partir de datos acústicos», RLA. Revista de Lingüística Teórica y Aplicada, 53 (2), II Sem, p. 13-45.

Fernández Planas A. M. a, Roseano P., Dorta J. y Martínez Celdrán E., 2013, «¿Continuidad prosódica en diferentes puntos de la Romania? El caso de algunas interrogativas», in Casanova E. y Calvo Rigual C. (eds.), Actas del 
XXVI Congreso Internacional de Lingüística i Filologia Románica, vol. I, Descripció històrica i/o sincrònica de las llengües romàniques: fonètica $i$ fonologia, Berlín, De Gruyter, p. 588-600.

Fernández Rei E., Moutinho L. C. y Coimbra R. L., 2014, «As entoacións galega e portuguesa: a fronteira á luz da dialectometría e da percepción», in Sousa X., Negro Romero M. y Álvarez R. (eds.), Lingua e identidade na fronteira galegoportuguesa, Santiago de Compostela, Consello da Cultura Galega, p. 115-141. Interlandi G. M., Romano A., Moutinho L. C. y Coimbra R. L., 2007, «Crosslinguistic Prosodic Variation in two Romance Languages: Portuguese and Italian», in González González M., Fernández Rei E., González Rei B. (eds.), Actas del III Congreso Internacional de Fonética Experimental, Santiago de Compostela, Xunta de Galicia, p. 377-387.

Jamet M.-C., 2007, «La transparence sonore du lexique», in Capucho F., Martins A. A. P., Degache C. y Tost M. (coord.), Dialogos em Intercompreensão, Lisboa, Universidade Católica Editora, p. 333-355.

López Bobo M. J., Muñiz Cachón C., Díaz Gómez L., Corral Blanco N., Brezmes Alonso D. y Alvarellos Pedrero M., 2007, «Análisis y representación de la entonación. Replanteamiento metodológico en el marco del proyecto AMPER», in Dorta, J. (ed.), La prosodia en el ámbito lingüístico románico, Madrid-Santa Cruz de Tenerife, La Página Ediciones, «Colección Universidad», p. 17-34.

Mairano P. (ed.), 2011, Intonations Romanes. Géolinguistique, hors-série 4, Grenoble, «Ellug».

Martín Gómez J. A., Dorta Luis J. y Sensui H., 2017, «Dificultades en el reconocimiento de patrones entonativos por parte de estudiantes de ELE», Porta Linguarum. Revista internacional de didáctica de las lenguas extranjeras, Monográfico II, p. 9-26.

Martínez Celdrán E., Fernández Planas A. M. a , Dorta Luis J. y Fernández Rei E., 2006, «La intercomprensió d'interrogatives absolutes amb tonemes descendents del català, de l'espanyol de Canàries i del gallec», Estudis Romànics, XXVIII, p. 7-28.

Martínez Celdrán E., Fernández Planas A. M. ${ }^{a}$, Dorta Luis J. y Fernández Rei E., 2007, «Reconocimiento de variedades lingüísticas a partir de la entonación: el caso de algunas interrogativas de Tenerife, Santiago de Compostela y Barcelona», Actas del III Congreso da Sociedade Española de Acústica Forense (SEAF), Santiago de Compostela, Ed. Xunta de Galicia, p. 225-247.

Mora E., Rojas N., Méndez J. y Martínez H., 2008, «Declarativas e interrogativas en el español venezolano. Percepción de la emisión con y sin contenido léxico», Language Design, Special Issue 2, p. 231-238.

Moutinho L. C., Coimbra R. L., Pereira Bendiha U., Romano A. y Contini M., 2004, «Estudo comparativo da variação prosódica em duas línguas românicas: o Português e o Italiano», in Freitas T. y Mendes A. (org.), Actas do XIX 
Encontro Nacional da Associação Portuguesa de Linguística, Lisboa, APL, p. 719-723.

Moutinho L. C., Coimbra R. L., Teixeira A. y Pereira M., 2005, «Variação entoacional em três áreas dialectais de Portugal Continental», in LAI, J-P. (ed.), Project AMPER Atlas multimédia prosodique de l'Espace roman Géolinguistique, Hors série no 3, p. 19-37.

Orta Gracia A., 2009, La enseñanza explícita de la pronunciación: creencias de los profesores y sus repercusiones en el aula de E/LE, Biblioteca Phonica, 9.

Rodrigues dos Santos G., Schurt Rauber A., Rato A., Cristina Kluge D. y Guilherme de Figueiredo M., 2013, «TP (v. 3.1): Una herramienta para experimentos de percepción», EFE, XXII, p. 335-366.

Romera Barrios L., Fernández Planas A. M. ${ }^{\mathrm{a}}$ y Salcioli Guidi V., 2009, "Análisis perceptivo de la entonación del castellano de Barcelona y del catalán de Barcelona», Estudios de Fonética Experimental, XVIII, p. 345-366.

Roseano P. y Fernández Planas A. M. a, 2013, «L'intonazione delle dichiarative neutre e delle interrogative polari in quattro varietà friulane: Agrons, Beivars, Tesis e Gradisca d'Isonzo», Ladinia, 37, p. 161-182.

Sensui H., 2015, «Un estudio fonético experimental sobre la percepción de la entonación de oraciones declarativas e interrogativas del español por hablantes nativos y estudiantes japoneses», Ph.D. dissertation, Sophia University, Tokyo.

Turculet A. y Bleortu C., 2013, «¿Hay una frontera prosódica entre las hablas de Transilvania y las de Moldavia?», Estudios de Fonética Experimental, XXII, p. 81-127.

Van Oosterzee C., 2005, «La percepció de l'entonació declarativa i interrogativa per part de parlants bilingües castellà-català i mono-lingües castellà de Barcelona», Estudios de Fonética Experimental, XIV, p. 295-307.

Zerling J-P. y Moutinho L. C., 2002, «Analyse comparée de trois patrons prosodiques en français et en portugais européen", Travaux de l'Institut de Phonétique de Strasbourg (TIPS), 32, p. 115-148. 